REVIEW ARTICLE

\title{
Midwifery in Canada
}

\author{
Karen Born*
}

\begin{abstract}
More drugs and technologies are now used in 'normal births' in North American than anywhere else in the world. This reflects in art the desire to master, conquer and control nature that was present among the colonist from the beginning (1)
\end{abstract}

Apparently the post-'60s, earth-mother garbage didn't go out with the love beads and bell bottoms. ... Things can go sour at any step in the birth process. That women continue to risk their babies' lives by buying into a selfgratifying scenario which lets them spin pretty fantasies about home births with incense burning is pretty scary (2).

Since childbirth is a core aspect of the human experience and a dramatic life cycle event, the practices associated with it can become quite contentious. The protest and celebration that accompanied the emergence and increasingly visible role of midwives in the Canadian health care system is understandable, considering the issues and context. The contradictory reactions of Canadians towards midwifery correlated with the many fears and misconceptions about the nature of birth, as well as the scope and role of midwives in the entire experience, from pregnancy to delivery and beyond. Many of these misconceptions influenced politics and policy makers, and entrenched oppositional view points of midwifery through powerful lobbies and interest groups. The historical forces and movements of the twentieth century, however, empowered the midwife lobby to propagate

* To whom correspondence should be addressed: Karen Born, 346 Lytton Blvd.Toronto, Ontario M5N 1R8, Canada. midwifery as a viable alternative to childbirth, stirred women to demand access to midwifery and enabled a greater acceptance of midwifery as a practice and profession within the health care system. Extensive studies and inquiries in the past thirty years suggest that midwifery may be a cost-effective, efficient alternative to obstetric, physician services.

Midwifery as Integrated into the Health Care System There are growing concerns that Canada is facing a crisis in maternity care because of looming shortages in professionals available to provide newborn and maternity care. The proportion of family physicians providing obstetric services has decreased from $36 \%$ in 1982 to $18 \%$ in 2000 (3). Moreover, due to increased lengths in postgraduate training and medical school enrollment limits, there has been a shortage of younger physicians, upon whom obstetrics traditionally relies for staffing (4). Midwives, as the only health professionals educated specifically to care for normal childbearing and newborns, are prepared to alleviate the burden of physician shortages in the future. Canada's current health care reforms and visions of complete community health would be greatly enhanced by further integration of midwifery into family medicine and women's health. Canada is the last industrialized country to formally legalize midwifes as health care practitioners. In all other industrialized countries, with the exception of the United States (U.S.), most babies are delivered by professional midwifes who are integrated into the health care system (5). The World Health Organization defines a midwife as a person who is qualified to practice midwifery:

She is trained to give the necessary care and advice to women during pregnancy, labour and the post natal period, to conduct normal deliveries on her own responsibility, and to care for the newly born infant. At 
all times she must be able to recognize the signs of abnormal or potentially abnormal conditions which necessitate referral to a doctor, and to carry out emergency measures in the absence of medical help. She may practice in hospitals, health units or domiciliary services. In any one of these situations she has an important task in health education within the family and community. In some countries, her work extends into the fields of gynecology, family planning or child care (6).

Midwives, as the deliverers of primary care in low risk pregnancies, can alleviate some of the current pressures on obstetricians, hospitals, physicians and nurses through taking on more cases, and developing collaborative relationships with other health care practitioners. In order for midwifery to become a legal, cost effective, and medically-sound practice uniformly across Canada, legal recognition, standardization of education, and funding would be required.

\section{A History of Midwifery in Canada: Practice on the Periphery}

The trajectory of midwifery in Canada reveals how the practice was marginalized and regenerated due to various influences, including the medical profession, state-imposed legal regulations and changes in cultural perspectives of childbirth. Midwifery was formally legislated out of medical practice in 1895 when the parliament, under pressure from the physician lobby, passed a law placing childbirth under the sole jurisdiction of physicians (7). Midwifery had been particularly prevalent in smaller, remote and indigenous communities, however, it became increasingly difficult for midwives to maintain the profession's viability due to this legislation. Midwives left the profession in fear of legal prosecution, and hence the technical skills and culture of midwifery was not transmitted to the next generation, though in some districts of Canada, the profession of the nurse-midwife began to form to counter the decline of traditional midwifery (8). Furthermore, the advent of insured medicine in Canada reinforced the abandonment of the midwife profession, due to a lack of clients and social support, as most Canadian women knew of no alternatives to birth attended by a physician. The absence of formalized midwifery-training programs, coupled with the dwindling number of practicing midwives, forced this once-mainstream practice to the periphery of health care.

Midwifery persisted during these difficult years in a few, isolated communities because of socio-cultural, geographical and historical factors. Many of these communities where midwifery was practiced lacked access to physicians and hospital facilities. This occurred mostly in isolated Northern and rural regions, such as Rankin Inlet in Northwest Territories. Unlike the rest of Canada, where maternal and infant care was and still is under provincial legislation, midwifery was self-regulated in these indigenous communities (7).

The marginalization of midwifery by the Canadian political and medical mainstream was a manifestation of the aversion towards midwifery by the Canadian health care establishment. The Canadian Medical Association, as well as Provincial Colleges of Physicians and Surgeons, opposed midwifery, citing studies deeming it inferior and unsafe in comparison to medicine's approach to childbirth. They strongly opposed homebirth, which they asserted constituted an inherent, unavoidable risk, and punitive measures were imposed on physicians in Alberta and Ontario to deter them from attending home births or providing backup support for planned home births $(9,11)$. The Canadian Nurses Association, though not as oppositional, was quite cautious since it perceived midwifery as encroaching on nursing interests and jurisdiction (7). Some Canadian nurses advocated an increased role for nursing professionals in maternal care, citing the U.S. model of the nurse-midwife as being amenable to their potential role in the Canadian system (7).

Historical currents during the World Wars slowly fostered resurgence in interest and support for midwifery in urban areas and in the political arena, as groups of women organized for increased access to midwifery, since many male physicians were on the front (8). This small lobby persisted and was strengthened exponentially with the advent of feminism and advances in perceptions of women's health. Despite the wider acceptance of midwifery, there was no standardization of midwife training and education in that era. Midwifery was a private practice, remunerated directly by the patient. Standards governing midwifery were not clearly defined and the practice was still considered outside of the usual legal modalities of health care.

Acquiescing to this lobby, government commissions to investigate midwifery were initiated in the late 1960 s and 1970s in order to determine a possible role for nurse-midwives in both urban and rural settings. These committees were often politically motivated, and an opportunistic method for governments to consolidate votes and support from women and feminists. The Committee on Healing Arts, set up in 1966 at the advent of universal medical care insurance in Ontario, recommended the integration of the nurse-midwife into the health care system (8). However, there was significant pressure by nursing associations, which did not wish to incorporate midwifery into their practice. These nursing professional groups suggested that 
Canada adopt the British model of midwifery as an independent and separate profession (8).

Societal demands for homebirth and natural births flowed from the counter-cultural center of CanadaBritish Columbia-in the 1970s. Midwifery became increasingly prevalent but was strictly relegated to the home setting. Midwives practicing at that time faced potential liability as they were providing medical care and services without formal recognition or uniform standards, and were thus vulnerable to the legal charge of practicing medicine without a license (8). Subsequent high profile trials led to increased attention to this issue, both by medical practitioners and the public, and fostered a number of government inquiries into the practice. This attention aided in the genesis of provincial midwifery coalitions and organizations to lobby the government and represent the position of midwives in several provinces.

A high profile death in 1985 of an Ontario baby, delivered at home by a midwife, brought the issue to public debate, media attention and scrutiny (8). The Crown was supported by medical professionals, who blamed the midwife for the death, whereas the midwife's defense was that the death was unavoidable. Both sides, nonetheless, concluded that regulation of midwifery was needed in Ontario. The trial and subsequent inquests evolved into a public inquiry into the state of midwifery in Ontario, and was crucial to the genesis of the Midwifery Task Force of Ontario (MTFO). The MFTO advocated the implementation of midwifery as a self-regulating profession with its own college, independent of both medicine and nursing. The MTFO also recommended that midwives have the option to practice in the home, or in an institutional setting, and that a nursing background should not be a prerequisite to midwifery education (8). When this legislation passed in Ontario in the early 1990s, and midwifery became a service reimbursed by the government, Canada joined the ranks of other countries that had already accepted midwifery and integrated the practice into their health care system (10). Government support for the midwifery initiatives were forthcoming for two main reasons: i) midwifery was seen as a cost effective form of care, and ii) midwifery support positioned governments as publicly supporting women's issues and promoting women's rights (8). Governments in British Columbia, Manitoba, Ontario and Quebec followed quickly and implemented midwifery-oriented health policy including legalization of the profession, standardization of training and fees remunerated through the public, provincial insurance plan. However, Saskatchewan and the Atlantic provinces have not legalized nor formalized midwifery practice at this time.
Policy and Practice of Midwifery in Canada

Despite the legalization of midwifery in some provinces, controversy persists in the medical community about the medical efficacy of midwife care, and there are disagreements about who provides the least expensive care to women with low risk pregnancy. In terms of optimal care for low risk pregnancy and normal vaginal delivery, studies have shown nearly congruent mortality and morbidity rates whether attended by midwives or physicians (12). In a study by Janssen et al. labour interventions in comparable midwife-attended homebirth births, midwife-attended hospital births and physician-attended hospital births were analyzed. The study showed that the home birth group displayed less frequent use of analgesia, electronic fetal monitoring, augmentation or induction of labour, and episiotomy, as well as fewer cesarian sections among women in the home birth group (6.4\%) compared with the midwife hospital group (11.9\%) and the physician hospital group (18.2\%) (12). Obstetricians are trained with a surgical orientation and some have a tendency to utilize interventional techniques to speed up labour (12).

The majority of births are low risk and can proceed without interventional, surgical techniques (12i). Therefore, midwives are critical of this surgical medical training and practice, though necessary in complicated births, for its application in low-risk births (1). The methodologies of teaching birth in medical school, and the charge that students and residents rarely witness a normal, spontaneous, unanaesthetized birth are some of the more extreme criticisms by midwives of the medical establishment (1).

The midwifery lobby advocates an increased role for themselves in natural, uncomplicated, spontaneous birth. As defined by the Ontario Midwifery Act in 1991, "the practice of midwifery is the assessment and monitoring of women during pregnancy, labour and the postpartum period and of their newborn babies, the provision of care during normal pregnancy, labour and postpartum period and the conducting of spontaneous normal vaginal deliveries."(13) Midwife advocates assert that midwifery training includes the ability to foresee complications and appropriately call for physician assistance. These precautions reduce potential problems and encourage referral for complicated births to obstetric specialists. The differing courses of treatment of medical and midwife professionals for uncomplicated birth explain the different approaches to care and methodologies employed. The obstetrician tends to see the patient for short checkups preceding birth, and then will attend to the woman while she is giving birth for short periods of time in a hospital, 
assisted by nurses and working in shifts. The midwife, however, develops a more consultative, collaborative relationship with the woman in the weeks leading up to birth, coaches the woman through the duration of delivery, and gives continued, postpartum maternal and newborn consultation.

Policy perspectives regarding cost efficiency often cite midwifery as a less costly alternative to physician care (11). There are a number of important variables that must be applied to fully balance the cost comparisons of midwife and physician deliveries. These include the reduced complications in midwifeattended births, the absence of technical equipment and drugs, and less support staff. Moreover, the midwifery option is often advantageous from a subjective client perspective, as their birth experiences are generally quite positive (11). A study by Harvey et al. confirmed this by indicating "women experiencing low risk pregnancies were more satisfied with care by midwives that with care provided by doctors." (14) Physician shortages, particularly of obstetricians and family physicians trained to provide obstetrical care, has been a major concern to decision makers since the mid-1990's. Many family physicians (who traditionally delivered babies in non-urban areas) decline or withdraw from obstetric practice due to fears of litigation, insufficient training or lifestyle concerns (3). The inclusion of midwives as the providers of primary maternal and newborn care has compensated for obstetrician and physician shortages in rural regions.

However, midwives are not universally welcomed by obstetricians, physicians and hospitals since midwives deal primarily with uncomplicated cases, which siphon billings of easier, quicker deliveries from physicians, relegating more difficult, intense, and problematic patients to physicians. This is known as 'creamskimming' or 'cherry picking.' Home birth implies less income to hospitals, and since they require a certain level of funds to properly care for complicated births that generate massive expenses of personnel time, and resources, this is seen as a problem. The issue of 'cream skimming' has not yet become critical in the midwifery debate, however, with the anticipated growth of midwifery, it will become an increasingly contentious issue in terms of allocation of government funds. Physicians who deal predominantly with complex cases confront premature personal burn-out and also reduced financial income and billings. These are among the main factors cited by physicians and hospital lobby groups to oppose greater roles for midwifery. Nevertheless, as policies evolve, there has been more impetus to include midwives in group practices to compensate for physician shortages and hospital overcrowding.

Trained, regulated and integrated midwives can potentially decrease stress on family physicians and obstetricians by attending the large number of uncomplicated births and counseling patients on mothering and lifestyle. Midwifery similarly addresses issues of determinants of health and focuses on health promotion by discussing nutrition, early breastfeeding and child care as part of the midwife repertoire of a dynamic patient-based approach focusing on interpersonal relationships and continuity of care (3).

\section{Provincial Perspectives: Midwifery Policy in Ontario and Alberta}

Much debate, particularly between different interest groups of health care practitioners and policymakers, has accompanied the implementation of midwifery into the health care system. Since each province has autonomy in health care, midwifery has been legalized and promoted to different degrees, reflecting the unique social, political and economic needs and citizen demands of each province. A comparison of the course of legalization of midwifery in Ontario and Alberta illustrates how these differences are accommodated and reflected in law. Policies of legalization and recognition tend to address midwife-training, ranges of responsibility, methods of payment, degree of autonomy and relations to other health care providers and institutions (16).

\section{Ontario}

The Ontario Midwifery Act of 1993 regulated midwifery as an autonomous health profession, established university programs for midwifery training, created the regulating body of the College of Midwives of Ontario, and additionally organized a system of provincial financial billing for midwifery services. This legislation established that midwives could be the primary practitioners of care, with the responsibilities of admission, direction of care, and discharge, and that midwives can practice at homes, hospitals, or birthing centers. This option of delivery of care validated midwifery and allowed midwives in Ontario to become increasingly familiar with other health professionals and participate more actively in research, education and policymaking regarding maternal and newborn care (17).

The practice of midwifery is growing in Ontario. There are three provincial universities (McMaster, Ryerson Polytechnic and Laurentian), which offer the Ontario Midwifery Training Programme, a four-year degree including intensive clinical exposure and training. There is also the College of Midwives, which 
offers the Prior Learning and Assessment Program. These programmes register approximately thirty-five new midwives each year (17). Arguably, this progressive policy and strong government support has facilitated the remarkable success and growth of midwifery in Ontario, with nearly 3,800 births in 1999 attended by midwives, and an anticipated 12,000 births in 2004 (10). The Ontario legislation is the foundation upon which the four provinces that subsequently legalized midwifery look to for precedent and policy directives. Patients increasingly demand midwifery, and midwives are anticipated to deliver $30 \%$ of all babies in Ontario and British Columbia by 2020 (10). Physicians in some regions have maintained strong efforts to retain their labour and delivery caseloads, and often the admission privileges of midwives are not respected (9). These physician interests are driven by various factors including a desire to preserve billing privileges and delivery priority. Midwives, however, are entrenched as part of the health care system spectrum, as their presence was implemented in an incremental and organized manner and the future evolution of the profession is assured through policy mechanisms facilitating greater patient choice and services.

\section{Alberta}

The legalization and inclusion of midwifery in Alberta differs dramatically from Ontario. The legislation granting legal recognition and professional status to midwifery does not allocate funds, educational initiatives or institutions for the growth of midwifery. The failure to pay midwives from the provincial budget, and trivial government support of midwifery led to a stagnation of development, and precipitated an exodus of trained midwives from Alberta despite recognition. The conservative fiscal stance of the Albertan government illustrates the power of government policy to facilitate or hinder shifts in health care. The government justified its actions by citing unnecessary competition between practitioners and "overlapping, nonexclusive scopes of practice" as specific criticisms of midwifery (9). Consequently, with no provisions to ensure that the supply of midwives would increase through education and training, Alberta experienced a crisis in midwifery. Widespread shortages of physicians delivering acute primary care, combined with the strong midwife and feminist lobby, enabled the politicization of midwifery, with the midwife lobby utilizing the argument of consumer choice to galvanize the public and induce government action in order to sustain the profession in the province (9). Midwifery remains politicized in Alberta, as it is currently part of the provincial government's initiative to reduce government health care costs through privatization of services of choice. That policy allows Albertans to have access to regulated midwifery and trained professionals, however, they must remunerate midwives directly for their services. Although women in Alberta can no choose to have a physician or midwife for their birth experience, only the physician fees are fully covered by Medicare. With midwife-attended births comprising $6.6 \%$ of all births in British Columbia, and $4.5 \%$ in Ontario, Alberta remains at the low rate of $1 \%$. In Alberta, the midwife-attended births occur mainly in the home and are only available to women who can afford this service. This discrepancy is directly attributed to a lack of government funds (18). This illustrates the decisiveness and impact of government support and policy, and how inclusion of midwifery services in the health care system facilitates greater access for women who choose such services.

\section{Midwifery and the Future of Health Care in Canada}

Midwifery is becoming increasingly important to future visions of health care in Canada, and is a strong political tool and issue to garner public interest and support. Despite its political salience, midwifery as a health care service and alternative to physician-assisted birth has garnered support for practical purposes so that the challenges and shortages of maternal care can be remedied by further integrating midwifery into the changing health care system as a cost effective, medically sound alternative to physician delivery. According to Monique Begin, the former Minister of Health and Welfare, the health care system must evolve to create health care providers who will be able to operate in the dynamic system of the future (19). This system must be increasingly responsive to patient needs, and linkages need to be forged between the various participants in the health care world, between traditional medicine, health promotion advocates, as well as the social and environmental determinants of health (19).

The model of care offered by midwifery is compatible with these principles, as midwifery is based upon standards such as continuity of care, informed choice and consent, and choice of birthplace. Midwives are not just trained to deliver babies, but rather to offer a range of care such as maternity care, breastfeeding instruction and support (19). The midwife-patient relationship is based upon trust, longevity and encouragement as midwifery mandates personalized, intimate care and relationships. Furthermore, within maternal care, midwifery acts as an interface between family physicians, specialists and other traditional modalities of health care.

The emphasis on group-based care within the 
midwifery community and the larger medical community is congruent with the current policy direction to group-based practices. Group-based care emphasizes comprehensiveness and cooperation, and seeks to alleviate pressures of physician shortages and waiting by offering around the clock care, referrals and attention (10). This projection of group practice is particularly significant in maternal and neonatal care within the medically underserviced urban, rural and remote populations and regions of Canada, since there has been a marked decrease in trained physicians able to provide maternal and neonatal care in these communities. Government policy in the last decade restricting medical school enrollments and residency training positions for obstetricians coupled with the decreasing numbers of trained family physicians are some factors that have created this shortage. In Ontario and British Columbia, where midwifery care has had adequate public funding, midwifery has been filling this widening gap for low risk obstetric services (10).

The loss of the basic maternal and neonatal care services has the potential to undermine the overall health of a community. Maternity and neonatal services are necessary for a community that wants to grow, and the development of a flexible, well-integrated community health care system with a wide variety of basic services is critical to serve the health care needs of the increasingly diverse population in Canada. For example, the presence of regulated midwifery in Rankin Inlet has resolved some social problems associated with moving an expectant mother away from her family and community in the weeks preceding and following birth (7).

The group-based practice answers the critical aspects of Begin's vision of the future of health care, as it is a clear and concerted effort towards professional interaction, linkages and collaboration. Furthermore, access is always a primary issue for midwives and the group-based practices provide access to a wider group of women, as their family physician will be able to refer low risk pregnancies to their midwife colleagues. The health care system, operating in the diverse urban, rural and remote regions of Canada, and accommodating the varied and complicated demands of the populace must be flexible, future-oriented and creative in order to accommodate the demands of the populace. The changing role of midwifery within Canadian health care, its ongoing, incremental acceptance into the mainstream and increasingly visible role as a policy driver illustrates how the system evolves to meet patient needs. Midwifery is poised to answer the challenges facing newborn and maternal care in Canada. However, Canadian citizens, health care professionals and decision makers must recognize this and nurture the development of this dynamic profession.

\section{REFERENCES}

1. The Boston Women's Health Book Collective. Our Bodies, Ourselves. New York: Simon and Schuster, 1998.

2. An Article in Calgary Herald, May 19, 1998 as quoted by Gunhild Hoogensen "The Politics of Birth: Midwifery in Alberta" 2000. www.birthpartnershipmidwives.com

3. Buske, Lynda. " A Crisis Aborning in Newborn and Maternity Care?" Canadian Medical Association Journal, March 6, 2001; 164 (5).

4. Chan, Benjamin TB "From Percieved Surplus to Percieved Shortage: What Happenned to Canada's Physician Workforce in the 1990's?" Canadian Institute for Health Information, June 2002.

5. Blais, Regis "Commentary/Commentaire: Are Home Births Safe?" Canadian Medical Association Journal, February 5, 2002; 166 (3).

6. Legislation Concerning Nursing/Midwifery Services and Education (EURO Reports and Studies, 1981) as quoted in Blais, Maheux, Lamber, Loiselle, Gauthier, Framarin "Midwifery defined by physicians, nurses and midwives: The birth of consensus?" Canadian Medical Association Journal 1994; 150 (5): 691-697.

7. Bailey, Lehr, Nicholas, Picco "Midwifery: Promotion and integration into Canada's healthcare system" Leadership in Health Services July/August 1993: 11-13.7.

8. Bourgeault, Ivy Lynn and Mary Fynes "Integrating Lay and Nurse Midwifery into the U.S. and Canadian Health Care Systems" Social Sciences \& Medicine"1997; 44 (7): 1051-1063.

9. McKendry, Rachael and Tom Langford "Legalized, regulated, but unfounded: midwifery's laborious professionalization in Alberta, Canada, 1975-99" Social Science \& Medicine, 2001;53: 531-542.

10. Canadian Midwifery Regulators Consortium, Submission to the Commission on the Future of Health Care in Canada "Regulated Midwifery and The Future of Health Care in Canada" 2001.

11. Reinharz D, Blais R, Fraser W, Constandriopolous AP "Costeffectiveness of midwifery services vs. medical services in Quebec, L'Equipe d'Evaluation des Projets-Pilotes SagesFemmes" Canadian Journal of Public Health 2000; 91(1): 112115 .

12. Janssen, P, Lee, SK, Ryan, E, Etches, D, Farquarson, D, Peacock,D and Klein, M. "Outcomes of planned home births versus planned hospital births after regulation of midwifery in British Columbia" Canadian Medical Association Journal 2002;166 (3):315-323.

12i. Low risk births can be identified at any stage of gestation, however in the Janssen et al study, 3.6\% of homebirths had to call for emergency transports in spite of the application of the following exclusion criteria including multiple birth, heart disease, hypertensive chronic renal disease, pregnancy induced hypertension with protein uria, insulin dependant diabetes, antepartum hemmorhage after 20 weeks gestation, active general herpes, breech or abnormal presentation, gestational age of less than 30 weeks or greater than 41 weeks at the onset of labour, more than one previous cesarian section, and mother transferred to hospital from another health care facility. (Janssen et al.)

13. www.aom.on.ca

14. Harvey,S, Rach, D, Stainton, MC, Jarrell, J, Brant, R. "Evaluation of Satisfaction of midwifery care" Midwifery 2002; 18: 260-267.

15. Ontario Association of Midwives (AOM) as quoted by Scott Piatkowski "Midwifery Remains a Safe Alternative", June 10, 2002. www straightgoods.ca

16. Blais, Maheux, Lamber, Loiselle, Gauthier, Framarin 
"Midwifery defined by physicians, nurses and midwives: The birth of consensus?" Canadian Medical Association Journal 1994; 150 (5): 691-697.

17. www.aom.on.ca/midwifery/NowandThen/html
18. www.asac.ab.ca

19. Bennett, Carolyn. Kill or Cure? Toronto: HarperCollins Publishers Ltd.,2000.

Karen Born is in her final year of a BA in Honours Political Science at McGill and will be graduating in 2004. Her areas of interest include Canadian health care reform, women in politics and peace and conflict studies. She plans to pursue either graduate studies or law after she finishes her degree. 